\title{
Síntesis, caracterización y aplicaciones de las nanopartículas de oro en tratamientos contra el cáncer
}

\section{Synthesis, characterization, and applications of gold nanoparticles in cancer treatments}

\author{
Iris C. López-Santillán ${ }^{a}$, Isaí Trejo-Teniente ${ }^{b}$, Elizabeth Hernández-Ortiz ${ }^{c}$,
} Blanca E. Jaramillo-Loranca ${ }^{d}$, Genaro Vargas-Hernández ${ }^{e}$, Maricela Villanueva-Ibáñez.

\begin{abstract}
:
Nowadays, cancer is one of the main causes of mortality and morbidity despite the many treatments available. For this reason, the search for novel treatments that do not negatively affect the quality of life of the patient continues. Gold nanoparticles are an alternative that combines diagnosis and treatment, the latter can be enhanced by using biological molecules to obtain them, as well as with conjugation with anticancer drugs.
\end{abstract}

Keywords:

Cancer, nanoparticles, green synthesis, diagnostic, treatment, pharmacodynamic.

Resumen:

Actualmente, el cáncer es una de las principales causas de mortalidad y morbilidad a pesar de los múltiples tratamientos disponibles. Por este motivo, continúa la búsqueda de tratamientos novedosos que no afecten negativamente a la calidad de vida del paciente. Las nanopartículas de oro son una alternativa que combina diagnóstico y tratamiento, este último se puede potenciar mediante el uso de moléculas biológicas para su obtención, así como con la conjugación con fármacos anticancerígenos.

\section{Palabras Clave:}

Cáncer, nanopartículas, síntesis verde, diagnóstico, tratamiento, farmacodinamia.

\section{Introducción}

El cáncer es una de las principales causas de muerte a nivel mundial, la OMS estima 18 millones de nuevos casos y más de 9.6 millones de muertes en 2018, aunque se espera un incremento de casos para la década de los 2030's hasta alcanzar los 22 millones. ${ }^{1-2}$
Los tipos de cáncer que se asociaron con los altos índices de mortalidad para 2018 fueron el de pulmón en primer lugar (1.8 millones), seguido del colorrectal (880 mil), estómago (783 mil), de hígado (782 mil) y mama ( 627 mil); esto a pesar de las múltiples terapias disponibles, ya que muchas de ellas presentan efectos secundarios y en

\footnotetext{
a Universidad Autónoma del Estado de Hidalgo, Instituto de Ciencias de la Salud, México; Universidad Politécnica de Pachuca, México, https://orcid.org/0000-0003-0740-1539, Email: iris_lopez6859@uaeh.edu.mx

b Universidad Politécnica de Pachuca, México, https://orcid.org/0000-0001-5728-652X, Email: isait@micorreo.upp.edu.mx

c Universidad Politécnica de Pachuca, México, https://orcid.org/0000-0002-5233-1018, Email: elibiotecnologia@micorreo.upp.edu.mx

d Universidad Politécnica de Pachuca, México, https://orcid.org/0000-0003-3812-5426, Email: bejarami@upp.edu.mx

e Universidad Politécnica de Pachuca, México, https://orcid.org/0000-0002-1931-2018, Email: ghernan@upp.edu.mx

Autor de Correspondencia, Universidad Politécnica de Pachuca, México, https://orcid.org/0000-0003-3209-8340, Email:
} 
especial la resistencia a fármacos, es por ello que se continúa con la búsqueda de terapias más efectivas. ${ }^{2-3}$

La nanotecnología es un campo muy prometedor para el desarrollo de productos novedosos en la medicina, sin embargo, actualmente son muy pocos los sistemas basados en nanotecnología que tienen un uso clínico. Las investigaciones recientes que enfatizan el uso de nanopartículas metálicas destacan las de oro, esto debido a sus propiedades relacionadas tanto con su tamaño nanométrico como con el método para su obtención. Cabe señalar que este último puede limitar su aplicación e impactar en su efecto anticancerígeno.

\section{Métodos de obtención.}

Los métodos para su obtención, comúnmente se dividen en métodos físicos y métodos químicos; en los primeros, mediante una fuente de energía, ya sea mecánica, calorífica o electromagnética, se obtienen nanopartículas de oro cerovalente "desnudas" por lo que se requiere, además, de agentes que garanticen su estabilidad en suspensión. Algunas de las técnicas de obtención de este tipo incluyen ablación láser, depósito en vapor químico, síntesis sonoquímica, entre otras. Por su parte, los métodos químicos incluyen, de forma general, aquellas síntesis que emplean como precursor una sal de oro y agentes reductores y estabilizantes, que en su mayoría son tóxicos, así como condiciones de reacción con un gasto importante de energía para alcanzar una temperatura y presión altas que, además, no aseguran mantener la estabilidad de las nanopartículas, especialmente cuando interactúan con moléculas y ambientes biológicos. Aunado a esto, algunas metodologías producen una amplia variedad de morfologías, lo que provoca bajos rendimientos al separar el tamaño y forma deseados. Entre las síntesis químicas más recurridas para la obtención de nanopartículas de oro se encuentra sol-gel, reducción fotoquímica y radioquímica. De esta manera, surgen alternativas de síntesis que proporcionan materiales nanométricos acordes con las necesidades, como es el caso de las biológicas. Estas síntesis toman agentes reductores y/o estabilizantes como proteínas, azúcares, polifenoles, catequinas, arubiginas o saponinas, de fuentes naturales como son las bacterias, hongos o plantas, incluyendo extractos o sus derivados, lo que convierte a este tipo de síntesis en un proceso ecoamigable y escalable. De igual forma, al emplear compuestos naturales, se asegura la biocompatibilidad y estabilidad en ambientes biológicos, además, la cubierta de agentes reductores brinda resistencia a la oxidación. ${ }^{1,4-8}$

\section{Caracterización}

Aún con las ventajas de la síntesis verde, es necesaria una caracterización exhaustiva para determinar las propiedades ópticas y fisicoquímicas de las nanopartículas, con el fin de definir su forma, tamaño y funcionalización, la cual es especialmente importante cuando se quiere unir con moléculas mediante algún grupo tiol, amino o ciano, para conferirle otras propiedades adicionales para su uso en terapias contra el cáncer. Cabe mencionar que, para ser aplicadas en terapia o tratamiento contra el cáncer, se prefiere una morfología esférica y los tamaños de partícula pueden variar desde $3 \mathrm{~nm}$ hasta 40 o $60 \mathrm{~nm}$, dependiendo del uso específico. La caracterización permite además una reproducibilidad del método, importante en el escalamiento. Algunas de técnicas de caracterización de nanopartículas de oro incluyen microscopía electrónica de barrido (MEB) y de transmisión (TEM) para conocer tamaño de partícula, morfología y estructura cristalina, esta última también se puede determinar mediante difracción de Rayos X. De igual modo, es importante conocer la funcionalización de las nanopartículas, ya sea conferida por el medio de síntesis o por adición posterior de algún ligando, mediante espectroscopía infrarroja por transformada de Fourier (FT-IR). Otras técnicas como dispersión dinámica de la luz (DLS), espectrofotometría ultravioleta visible (UV-Vis) y espectroscopia de fotoluminiscencia permiten de igual modo obtener información sobre las propiedades fisicoquímicas. ${ }^{1,4-8}$

\section{Aplicaciones}

En la aplicación de las nanopartículas de oro en la lucha contra el cáncer es posible seguir tres vías: la primera de ellas como diagnóstico, donde se emplean como agentes de contraste, para ello se induce láser $u$ ondas ultrasónicas que penetran los tejidos profundos y generan imágenes fotoacústicas. La segunda es en el uso directo de las mismas como tratamiento, para tal fin se requiere que sean citotóxicas o inductoras de apoptosis, de igual forma, por sus características fisicoquímicas, pueden ser empleadas en terapia fotodinámica, fototérmica y radioterapia, ya que pueden fungir como nanocalefactores cuando se irradia sobre ellas una longitud de onda específica. La tercera vía es como acarreadoras de moléculas para aumentar el efecto terapéutico, lo que facilita la destrucción del tumor y también la generación de especies reactivas de oxígeno (EROS) que desencadenan en muerte celular. ${ }^{1,4-8}$

Si bien las nanopartículas de oro han demostrado actividad anticancerígena, esta varía según la línea celular, por ello es necesario realizar análisis preclínicos en células tumorales in vitro, que evalúen la viabilidad celular y permitan determinar la citotoxicidad preliminar. 
Así mismo, se debe caracterizar la farmacodinamia al observar la integridad de la membrana celular, el método de internalización a partir de la química de la superficie de las nanopartículas, si se induce muerte celular a través de la fragmentación del ADN o mediante la generación de EROS y la vía que siguen (apoptosis o necrosis). También son requeridos estudios in vivo que permitan optimizar métodos más efectivos y biocompatibles, característica necesaria para una aplicación biomédica. ${ }^{1,4-8}$

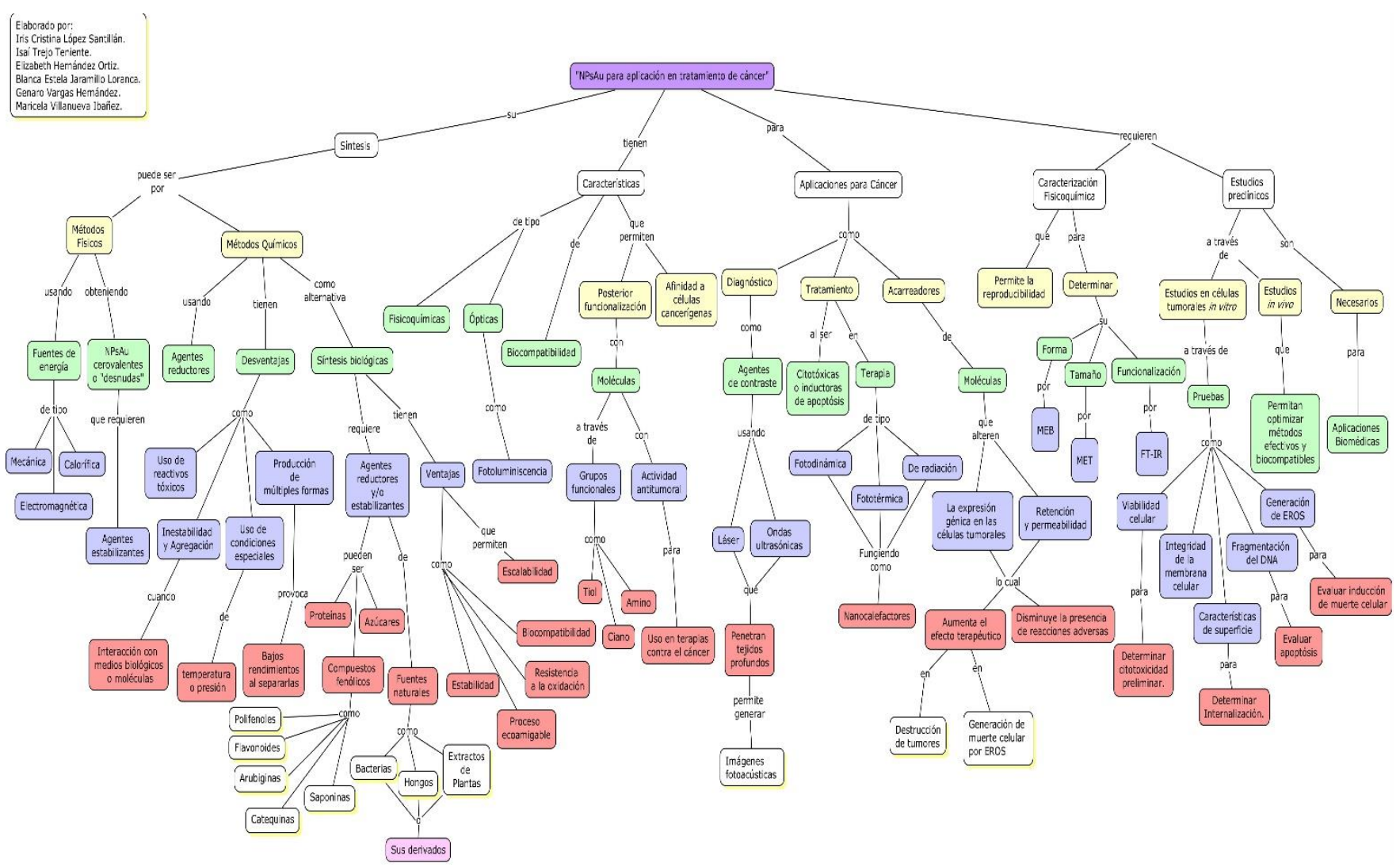

Figure 1. NPSAu para aplicación en tratamiento de cáncer

\section{Conclusiones}

En conclusión, es posible advertir que en el combate contra el cáncer las nanopartículas de oro son una alternativa prometedora para su detección y tratamiento, particularmente aquellas derivadas de métodos biológicos.

\section{Referencias}

[1] Rao PV, Nallappan D, Madhavi K, Rahman S, Jun Wei L, Gan SH. Phytochemicals and Biogenic Metallic Nanoparticles as Anticancer Oxidative Medicine and Cellular Longevity. 2016; 1-15.

[2] World Health Organization. (2018). Cancer. Recuperado de http/www.who.int/en/news-room/fact-sheets/detail/cancer
[3] International Agency for Research on Cancer. (2020). World Cancer Report: Cancer Research for Cancer prevention. Recuperado de https://www.iarc.who.int/featured-news/new-world-cancer-report/

[4] Khan I, Saeed K, Khan I. Nanoparticles: Properties, applications and toxicities. Arabian Journal of Chemistry. 2019; 12: 908-931.

[5] Amarnath K, Mathew NL, Nellore J, Siddarth CR, Kumar J. Facile synthesis of biocompatible gold nanoparticles from Vites vinefera and its cellular internalization against HBL-100 cells. Cancer nanotechnology. 2011; 2: 121-132.

[6] Gurunathan S, Han J, Park JH, Kim JH. A green chemistry approach for synthesizing biocompatible gold nanoparticles. Nanoscale research letters. 2014; 9: 248.

[7] Sztandera K, Gorzkiewicz M, Klajnert B. Gold Nanoparticles in Cancer Treatment. Molecular Pharmaceutics. 2019; 16: 1-23.

[8] Wang S. Lu G. Applications of Gold Nanoparticles in Cancer Imaging and Treatment. Noble and Precious Metals - Properties, Nanoscale Effects and Applications. IntechOpen, London. 2017; 291-308. 